\title{
ON A CERTAIN GROUP STRUCTURE FOR POLYNOMIALS
}

\author{
T. T. MOH $^{1}$
}

\begin{abstract}
A problem of C. C. Yang concerning level sets in complex analysis is generalized and algebraized to a problem about a group structure of polynomials. The group structure is established and several partial solutions of Yang's problem follow at once.
\end{abstract}

1. Introduction. In [1] C. C. Yang has raised the following interesting problem about level sets in complex analysis.

Problem 1. Let $p(z)$ and $q(z)$ be two polynomials of the same degree over the complex numbers $\mathbf{C}$ such that

$$
p(z)(p(z)-1)=0 \Leftrightarrow q(z)(q(z)-1)=0 .
$$

Prove (or disprove) that either $p \equiv q$ or $p+q \equiv 1$.

Let us complete the square. We have

$$
p(z)(p(z)-1)=\left(p(z)-\frac{1}{2}\right)^{2}-\frac{1}{4} .
$$

In fact the above problem is to determine if the zero set of a function $F=f^{2}-\frac{1}{4}$ completely determines the function $f$ up to \pm sign. We may assume that the function $F$ is monic and replace $\frac{1}{4}$ by any nonzero constant. Hence we have the following problem which is equivalent to Problem 1 .

Problem 1*. Let $F(z)=f^{2}-\alpha^{2}, G(z)=g^{2}-\beta^{2}$ be two nonconstant monic polynomials of the same degree with $\alpha, \beta$ nonzero constants. Furthermore

$$
F(z)=0 \Leftrightarrow G(z)=0 .
$$

Prove (or disprove) that $F(z) \equiv G(z)$.

We may raise the following "global" problem.

Problem 2. Given a set $S$. Determine all polynomials $F(z)=f^{2}-\alpha^{2}$ with $f$ monic, a nonzero constant and $F(z)$ does not vanish outside $S$.

An even more general problem follows.

Problem 3. Given a set $S$. Determine the algebraic structure of the set of polynomials $F(z)=f^{2}-\alpha^{2}$ with $f$ monic, $\alpha$ a polynomial $(f, \alpha)=1, \operatorname{deg} f-$ $\operatorname{deg} \alpha>a$ constant and $F(z)$ does not vanish outside $S$.

We give the following definition.

Received by the editors August 6, 1978 and, in revised form, May 1, 1980.

1980 Mathematics Subject Classification. Primary 12D05, 12D10

Key words and phrases. Level set, generator, rank, abelian group, Hurwitz formula.

${ }^{1}$ This work was partially supported by the National Science Foundation under grant no. 0029-501395, at Purdue University. 
Definition. Let $k$ be a field of characteristic $\neq 2$. Let $F=f^{2}-\alpha^{2}$ with $f, \alpha \in k[x]$ and $(f, \alpha)=1$. Let $F_{\alpha}$ denote the pair $(F, \alpha)$. Let $n>1$ and $P_{n}=\left\{F_{\alpha}\right.$ : $F$ and $f$ monic, $\operatorname{deg} f-\operatorname{deg} \alpha>n$ \}.

REMARK. In the above definition $F_{\alpha} \neq F_{-\alpha}$ if $\alpha \neq 0$.

Definition. Let $S$ be a set. Let $P_{n}(S)=\left\{F_{\alpha} \in P_{n}: F\right.$ has no zeros outside $\left.S\right\}$.

Definition. Let $F_{\alpha}=f^{2}-\alpha^{2}, G_{\beta}=g^{2}-\beta^{2}$. Let

$$
h=(f g+\alpha \beta, \beta f+\alpha g)
$$

be monic, then we shall define * as follows

$$
F_{\alpha} * G_{\beta}=\left[\frac{1}{h}(f g+\alpha \beta)\right]^{2}-\left[\frac{1}{h}(\beta f+\alpha g)\right]^{2} .
$$

Our main theorem is the following.

TheOREM. The group $\left(P_{n}, *\right)$ is the torsion free abelian group. Given any set $S$, $P_{n}(S)$ is a subgroup of $P_{n}$. Furthermore $P_{n}(S)=\{e\}$ if $\operatorname{card}(S)<n$ and $P_{n}(S)$ is of rank $<\operatorname{card}(S)-n$ if $n<\operatorname{card}(S)<\infty$ where $\operatorname{card}(S)$ denotes the cardinal number of the set $S$.

There are examples to indicate that the above theorem is the best possible. Some cases of Problem 1* can be answered at once by using the above theorem.

We wish to express our thanks to D. Drasin and A. Weitsman for showing us the problem of Yang and to W. Heinzer for helping us prove Lemma 5.

2. Proof of the Theorem. First we study the group structure of $\left(P_{n}, *\right)$.

LEMMA 1. $\left(P_{n}, *\right)$ is associative.

Proof. Let

Then

$$
\begin{gathered}
F_{\alpha}: F=f^{2}-\alpha^{2}, \\
G_{\beta}: G=g^{2}-\beta^{2}, \\
H_{\gamma}: H=h^{2}-\gamma^{2} .
\end{gathered}
$$

$$
\begin{aligned}
F \cdot G \cdot H & =(f g h+\beta \gamma f+\alpha \gamma g+\alpha \beta h)^{2}-(\alpha g h+\beta f h+\gamma f g+\alpha \beta \gamma)^{2} \\
& =p^{2}-\delta^{2} .
\end{aligned}
$$

Let $(p, \delta)=q$ be monic. Then $F *(G * H): U=u^{2}-\varepsilon^{2}$ and $(F * G) * H: U=$ $u^{2}-\varepsilon^{2}$, where $u=p / q, \varepsilon=\delta / q$.

LEMMA 2. $F_{\alpha} * F_{-\alpha}=e: 1=1^{2}-0^{2}$.

Proof. Trivial.

LeMMA 3. $F_{\alpha} * G_{\beta}=G_{\beta} * F_{\alpha}$.

Proof. Trivial.

LeMMA 4. Let $F_{\alpha}: F=f^{2}-\alpha^{2}$ and $\alpha_{m}=\sum_{i \text { odd }} C_{i}^{m} f^{m-i} \alpha^{i}$. Then

$$
\underbrace{F_{\alpha} * F_{\alpha} * \ldots * F_{\alpha}}_{m}=\left(F^{m}\right)_{\alpha_{m}} \text {. }
$$


Proof. The above statement is true for $m=1$. Let us assume it is true for $m$. Then we have

$$
\begin{aligned}
F^{m}= & \left(\sum_{j \text { even }} C_{j}^{m} f^{m-j} \alpha^{j}\right)^{2}-\left(\sum_{i \text { odd }} C_{i}^{m} f^{m-i} \alpha^{i}\right)^{2} \\
& \times\left(\sum_{j \text { even }} c_{j}^{m} f^{m-j} \alpha^{j}\right)^{f}-\left(\sum_{i \text { odd }} C_{i}^{m} f^{m-i} \alpha^{i}\right) \alpha \\
= & \sum_{j \text { even }} C_{j}^{m+1} f^{m+1-j} \alpha^{j}\left(\sum_{j \text { even }} C_{j}^{m} f^{m-j} \alpha^{j}\right) \alpha+\left(\sum_{i \text { odd }} C_{i}^{m} f^{m-i} \alpha^{i}\right) f \\
= & \sum_{i \text { odd }} C_{i}^{m+1} f^{m+1-i} \alpha^{i} .
\end{aligned}
$$

Moreover we have

$$
\sum_{j \text { even }} C_{j}^{m+1} f^{m+1-j} \alpha^{j} \pm \sum_{i \text { odd }} C_{i}^{m+1} f^{m+1-i} \alpha^{i}=(f \pm \alpha)^{m+1}
$$

Hence $\left(\Sigma_{j \text { even }} C_{j}^{m+1} f^{m+1-j} \alpha^{j}, \sum_{i \text { odd }} C_{i}^{m+1} f^{m+1-i} \alpha^{i}\right)=\left((f+\alpha)^{m+1},(f-\alpha)^{m+1}\right)=$ 1.

Thus $\left(F^{m}\right)_{\alpha_{m}} * F_{\alpha}=\left(F^{m+1}\right) \alpha_{m+1}$.

Combining Lemmas $1,2,3$, and 4 we conclude that $\left(P_{n}, *\right)$ is a torsion free abelian group. Clearly $P_{n}(S)$ is a subgroup of $P_{n}$.

LEMMA 5. If $\operatorname{card}(S)<n$ then $P_{n}(S)=\{e\}$.

Proof. We may assume $k$ algebraically closed. Then $[k(x): k(\alpha / f)]=m+n=$ $\operatorname{deg} f>\operatorname{deg} \alpha=m$. It follows from the Hurwitz formula that [2]

$$
-2=-2(n+m)+\sum(e(p)-1)
$$

where $p$ runs through all places of $k(x)$ and $e(p)$ is the ramification index of $p$.

We have $e(p)=n$ if $p=(x=\infty)$ and $\Sigma(e(p)-1)=2(n+m)-d$ for $p$ centered at $\alpha / f= \pm 1$ with $d$ the total number of district roots for $f \pm \alpha$. Hence we have $d \geqslant n+1$.

LeMMA 6. Let $F_{\alpha}: F=f^{2}-\alpha^{2}$ and $G_{\beta}: G=g^{2}-\beta^{2} \in P_{n}(S)$. Let $b \in S$ and let $F$ and $G$ have zeros of multiplicities $m$ and $l$, respectively, at $b$. Suppose $m \leqslant l$. Then $F_{\alpha} * G_{\beta}$ and $F_{-\alpha} * G_{\beta}$ have zeros of mutiplicities $m+l$ and $l-m$ at $b$ in some order.

PRoof. We assume without loss of generality that $(x-b)$ is a factor of $g-\beta$. Then

$$
\beta^{2} F-\alpha^{2} G=(\beta f+\alpha g)(\beta f-\alpha g) .
$$

Suppose $x-b$ is a factor of $f-\alpha$. Then $x-b$ is a factor of $\beta f-\alpha g$ with multiplicity $>\min (m, l)$ with equality if $m \neq l$. Hence

$$
(x-b)^{2 m} \mid\left(F \cdot G,(\beta f-\alpha g)^{2}\right) \text { and }(x-b)^{2 m+1} \mid\left(F \cdot G,(\beta f-\alpha g)^{2}\right) .
$$

Let $F_{-\alpha} * G_{\beta}=H_{\lambda}=h^{2}-\lambda^{2}$. Then $H$ has a zero of multiplicity $m-l$ at $b$. Since $x-b$ is a factor of $\beta f-\alpha g$, it cannot be a factor of $f+g$. Otherwise $x-b$ would 
be a factor of $2 \beta f$ and $2 \alpha g$. If it is a factor of $F$ (i.e. $m>0$ ) then it cannot be a factor of either $f$ or $\alpha$. Hence it must be a factor of $g$ and $\beta$, a contradiction. We conclude that

$$
(x-b) \mid(F \cdot G,(\beta f+\alpha g)) .
$$

Let $F_{\alpha} * G_{\beta}=U_{e}: U=u^{2}-\varepsilon^{2}$. Then $U$ has multiplicity $m+l$ at $b$.

Suppose $x-b$ is a factor $f+\alpha$. Then replace $\alpha$ by $-\alpha$ in the above argument. Q.E.D.

LeMma 7. Suppose $F_{\alpha}: F=f^{2}-\alpha^{2} \in P_{n}(S)$ where $F$ has a zero of minimal positive multiplicity $m$ at $b \in S$. Let $G_{\beta}: G=g^{2}-\beta^{2} \in P_{n}(S)$ with multiplicity $l$ at $b \in S$. Then $l=m \cdot p$ for some integer $p$. Furthermore either $\left(F^{p}\right)_{\alpha_{p}} * G$ or $\left(F^{p}\right)_{(-\alpha)_{p}} * G$ does not vanish at $b$.

Proof. Follows from Lemma 6 by Euclidean algorithm.

Proof of The LAST PART OF TheOrem 1. If there is no element in $\boldsymbol{P}_{n}(S)$ with positive multiplicity at $b \in S$ then $P_{n}(S)=P_{n}(S \backslash\{b\})$. The proof is completed by induction on the cardinality of $S$. Otherwise choose $F_{\alpha}: F=f^{2}-\alpha^{2}$ with smallest possible positive multiplicity. Then $P_{n}(S)$ is generated by $F_{\alpha}$ and $P_{n}(S \backslash\{b\})$.

\section{Discussion.}

Proposition 1. Let $F_{\alpha} \in P_{n}$. If $F=G^{l}$ as ordinary power with $G$ monic, then $G_{\beta} \in P_{n}$ for some $\beta$.

Proof. Let $F=f^{2}-\alpha^{2}=(f-\alpha)(f+\alpha)=G^{l}$. Since $(f-\alpha, f+\alpha)=1$, then we have

$$
f-\alpha=h_{1}^{l}, \quad f+\alpha=h_{2}^{l},
$$

with $\left(h_{1}, h_{2}\right)=1$ and $h_{1}, h_{2}$ are monic. We have

$$
2 \alpha=\Pi\left(h_{2}-w^{i} h_{1}\right)
$$

where $w$ is a primitive $l$ th root of unity. Let

$$
\operatorname{deg} h_{1}=\operatorname{deg} h_{2}=c, \quad \operatorname{deg}\left(h_{2}-h_{1}\right)=e .
$$

Then we have

$$
\begin{gathered}
\operatorname{deg}\left(h_{1}+h_{2}\right)=c, \quad \operatorname{deg} \alpha=(l-1) c+e, \\
\operatorname{deg} f=l c, \quad n=l c-[(l-1) c+e]=c-e,
\end{gathered}
$$

and

$$
G=h_{1} h_{2}=\left[\frac{1}{2}\left(h_{1}+h_{2}\right)\right]^{2}-\left[\frac{1}{2}\left(h_{1}-h_{2}\right)\right]^{2} .
$$

Choose $\beta=\frac{1}{2}\left(h_{1}-h_{2}\right)$.

For Yang's problem the following Propositions 2 and 3 establish partial solutions.

Proposition 2. If card $S=n+1$, then $P_{n}(S)$ is a cyclic group of one generator. Hence the answer to Problem 1 in this case is affirmative.

Proof. It follows trivially from our theorem. 
Proposition 3. If card $S=2 n-1$, then there is at most one polynomial $F$ with $\operatorname{deg} F=2 n, F$ vanishes and only vanishes at every point of $S$ and $F_{\alpha} \in P_{n}(S)$ for some $\alpha$.

Proof. Let $G$ : $G=g^{2}-\beta^{2}$ be another one. Then $F_{\alpha} * G_{\beta} \neq\{e\}=F_{-\alpha} * G_{\beta}$, since $(\beta f+\alpha g, \beta f-\alpha g, F G)=1$ and we have

$$
\begin{aligned}
F G & =(f g+\alpha \beta)^{2}-(\beta f+\alpha g)^{2} \\
& =(f g-\alpha \beta)^{2}-(\beta f-\alpha g)^{2}, \\
\beta^{2} F-\alpha^{2} G & =(\beta f+\alpha g)(\beta f-\alpha g) .
\end{aligned}
$$

Suppose $S=\left\{b_{1}, \ldots, b_{2 n-1}\right\}$ with $\left(x-b_{1}\right) F=\left(x-b_{2}\right) G$. Then at least one of the two factors $\beta f+\alpha g$ and $\beta-\alpha g$ must have at least $n-1$ of the remaining points as roots. Thus either $F_{\alpha} * G_{\beta}$ or $F_{-\alpha} * G_{\beta}$ will have at most $n$ district roots which contradicts Lemma 5.

We shall complete our article by listing several examples.

EXAMPLE 1. The rank of $P_{1}(S)=\operatorname{card}(S)-1$. Let $S=\left\{b_{1}, \ldots, b_{n}\right\}$. Then $F_{\alpha_{1}}=\left(x-b_{1}\right)\left(x-b_{i}\right)=\left[x-\frac{1}{2}\left(b_{1}+b_{i}\right)\right]^{2}-\left[\frac{1}{2}\left(b_{1}-b_{i} d\right)\right]^{2}$ for $i=2, \ldots, n$ clearly generate $P_{1}(S)$.

EXAMPLE 2. Let

$$
S=\left\{-\frac{1}{2}, 0,1, \frac{3}{2}\right\}, \quad F_{\alpha}: x^{2}\left(x-\frac{3}{2}\right)(x-1)^{2}\left(x+\frac{1}{2}\right)=\left(x^{3}-\frac{3}{2} x^{2}+\frac{1}{4}\right)^{2}-\left(\frac{1}{4}\right)^{2}
$$

and

$$
G_{\beta}: x\left(x-\frac{3}{2}\right)(x-1)\left(x+\frac{1}{2}\right)=\left(x^{3}-x-\frac{3}{8}\right)^{2}-\left(\frac{3}{8}\right)^{2} .
$$

Then $F_{\alpha}$ and $G_{\beta}$ generate $P_{2}(S)$ with rank $P_{2}(S)=2$.

\section{REFERENCES}

1. Complex analysis, Proceedings of the SUNY Brockport Conference, Dekker, New York and Basel, 1978, p. 169.

2. W. Fulton, Algebraic curves, Benjamin, New York, 1969.

Department of Mathematics, Purdue University, West Lafayette, Indina 47907 